\title{
Emulating Realistic Bidirectional Spatial Channels for MIMO OTA Testing
}

\author{
Wei Fan, ${ }^{1}$ Pekka Kyösti, ${ }^{2}$ Jesper $\emptyset$. Nielsen, ${ }^{1}$ Lassi Hentilä, ${ }^{2}$ and Gert F. Pedersen ${ }^{1}$ \\ ${ }^{1}$ Department of Electronic Systems, Faculty of Engineering and Science, Aalborg University, 9220 Aalborg, Denmark \\ ${ }^{2}$ Anite Telecoms Oy, 90230 Oulu, Finland \\ Correspondence should be addressed to Wei Fan; wfa@es.aau.dk
}

Received 27 August 2015; Accepted 9 November 2015

Academic Editor: Mugen Peng

Copyright (c) 2015 Wei Fan et al. This is an open access article distributed under the Creative Commons Attribution License, which permits unrestricted use, distribution, and reproduction in any medium, provided the original work is properly cited.

\begin{abstract}
This paper discusses over the air (OTA) testing for multiple input multiple output (MIMO) capable terminals with emphasis on modeling bidirectional spatial channel models in multiprobe anechoic chamber (MPAC) setups. In the literature, work on this topic has been mainly focused on how to emulate downlink channel models, whereas uplink channel is often modeled as free space line-of-sight channel without fading. Modeling realistic bidirectional (i.e., both uplink and downlink) propagation environments is essential to evaluate any bidirectional communication systems. There have been works stressing the importance of emulating full bidirectional channel and proposing possible directions to implement uplink channels in the literature. Nevertheless, there is no currently published work reporting an experimental validation of such concepts. In this paper, a general framework to emulate bidirectional channels for time division duplexing (TDD) and frequency division duplexing (FDD) communication systems is proposed. The proposed technique works for MPAC setups with arbitrary uplink and downlink probe configurations, that is, possibly different probe configurations (e.g., number of probes or their configurations) in the uplink and downlink. The simulation results are further supported by measurements in a practical MPAC setup. The proposed algorithm is shown to be a valid method to emulate bidirectional spatial channel models.
\end{abstract}

\section{Introduction}

Multiple-input multiple-output (MIMO) systems have demonstrated their capabilities to improve system capacity and link reliability in rich multipath environments [1]. The propagation channel and antenna design are the two key parameters that together impact MIMO system performance [2]. Over the air (OTA) testing of MIMO and diversity capable terminals has attracted great research attention, since OTA testing is the only way to evaluate the radio in a realistic way [3]. Several different OTA systems have been proposed to evaluate MIMO capable terminals, for example, the two stage systems (with the conducted/radiated option) $[4,5]$, reverberation chamber based systems (with/without a radio channel emulator) $[6,7]$, and multiprobe anechoic chamber (MPAC) systems (with two-dimensional (2D)/3D probe configurations) $[8,9]$. The MPAC system is a promising candidate, due to its capability to physically reproduce arbitrary multipath environments in the laboratory.
Research work in MPAC setups has been focused on emulating downlink (i.e., communication from base station (BS) to mobile station (MS)) channel models, where the uplink (i.e., communication from MS to BS) channel is often realized through a single communication antenna and a cable [3]. As shown in Figure 1, the uplink signal is radiated by the device under test (DUT) and received by an uplink communication antenna inside the anechoic chamber. Then the uplink antenna is directly connected to the input of the BS emulator with a cable, and therefore the uplink channel is constant and does not experience any fading. However, modeling uplink channel as a static free space line-of-sight (LOS) is not realistic.

Emulating realistic bidirectional channels (i.e., both uplink and downlink) is gaining significant research interest, as it is useful for evaluating any bidirectional radio communication system [10]. Bidirectional channel emulation is mandatory, especially for device testing in multiuser interference scenarios and cognitive radio terminals testing 


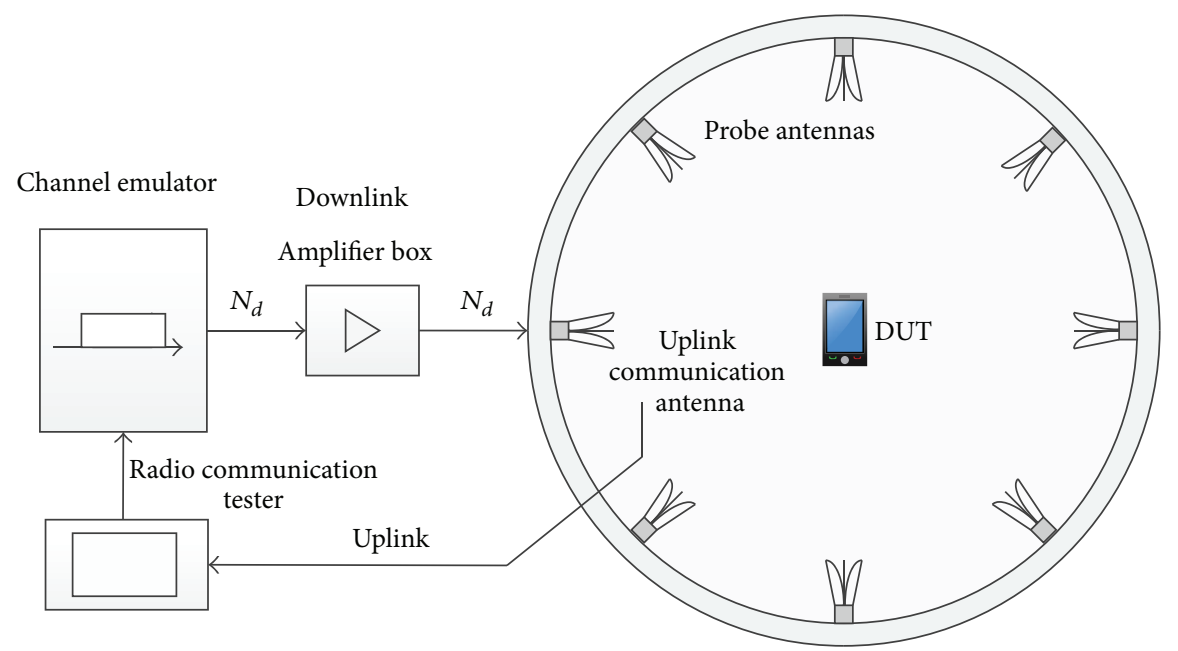

FIgURE 1: An illustration of MPAC setups in the standards. $N_{d}$ is the number of probes used for the downlink. The probes and the channel emulator are used to emulate downlink channel models. As for the uplink, a cable feeding directly to the radio communication tester is used and therefore fading of uplink channel is not emulated.

$[10,11]$. The uplink channel state information can be critical for downlink performance in some closed loop communication systems with adaptive modulation, coding, and MIMO transmission modes. In the time-division duplexing (TDD) mode, as the downlink and uplink propagation channels are reciprocal, the BS may estimate the radio channel state information from the uplink channel. Then the information is utilized in selecting downlink transmission mode or even in weighting $\mathrm{Tx}$ antenna signals. Also in the frequencydivision duplex (FDD) case, it may be important to perform testing with realistic uplink fading, for example, in order to get typical feedback channel errors. Furthermore, to reduce the difference in peak data rate between uplink and downlink, uplink with up to 4 transmission antennas (i.e., 4 transmission antennas on MS) has been introduced in LTE-Advanced (Release 10 and beyond) [12]. Furthermore, as discussed in [13], the terms downlink and uplink are not relevant to communication scenarios utilizing ad hoc and mesh networks. It would be desirable to model realistic bidirectional propagation environments to evaluate the MIMO terminal performance.

Very few papers have addressed emulation of bidirectional channel models in MPAC setups. The concept of emulating bidirectional channel models for TDD systems in MPAC setups was briefly mentioned in [14], though no details were given. In $[10,11,13]$, the importance of bidirectional channel emulation is well described for closed-loop OTA testing, OTA testing of cognitive radio terminals, and OTA testing in car to car/infrastructure scenarios. In [10], it was proposed to apply wave field synthesis principles to generate uplink channel models, though no results were repoted to validate the proposal. The main contributions of this paper lie in the following aspects:

(i) It is often assumed that frequency separation of two different carrier frequencies is sufficiently large compared with the channel coherence bandwidth, and therefore fading characteristics for the frequencyseparated channels are uncorrelated. This is not always correct, as demonstrated in this work using data from a channel measurement campaign. The knowledge gained in the measurements is then adopted to emulate bidirectional channels in MPAC setups for FDD systems.

(ii) A general framework to emulate bidirectional channels both for TDD and FDD communication systems is proposed. The proposed technique works for MPAC setups with different uplink and downlink probe configurations.

(iii) Several multiprobe anechoic chamber setups, capable of emulating realistic bidirectional channels, are proposed and evaluated. The proposed technique is demonstrated by simulation results for some representative channel models.

(iv) To validate the proposed algorithm, the algorithm has been implemented in a commercial channel emulator and validated in a practical MPAC setup. A measurement setup for validation purpose is proposed in the paper as well. To the best of the authors knowledge, it is the first time that bidirectional channel models have been emulated in a practical MPAC setup for MIMO OTA testing.

\section{Requirements on Uplink Channel Modeling}

The target channel models adopted for the downlink in MIMO OTA testing are the well-accepted and standardized geometry-based stochastic channel (GBSC) models [3, 15]. MIMO channel models like SCME, WINNER, and IMTAdvanced models belong to this family $[16,17]$. Geometrybased modeling enables separation of channel propagation and antennas at the transmitter ( $\mathrm{Tx})$ and receiver $(\mathrm{Rx})$ side. A GBSC model consists of multiple clusters (i.e., groups of 
multipath components), each of which is modeled by its cluster power, delay, nominal angle of arrival (AoA), nominal angle of departure (AoD), angle spread of arrival (ASA), angle spread of departure (ASD), and cross polarization power ratio (XPR). The GBSC models are used to model the uplink channels in the paper as well, to be aligned with the downlink channels.

In TDD communications systems, as the same frequencies are utilized for the downlink and uplink, the uplink and downlink channels are considered as reciprocal. In the FDD communication systems, the uplink and downlink channels are frequency separated. In this section, a literature review on cross-correlation of channel parameters between frequency separated channels is firstly given.

2.1. Cross-Correlation between Frequency Separated Channels. The correlation properties between frequency separated channels have attracted research attention, as they are important to the design and performance prediction of multicarrier communications [18].

2.1.1. Large Scale and Small Scale Channel Parameters. The same terminologies as in GBSC models are adopted in this paper. Large and small scale parameters are specified to generate channel coefficients [19]. Large scale channel parameters, which include shadow fading, angle spread, delay spread, and Rician factor, describe GBSC models at a macroscopic level [19]. Small scale parameters, that is, cluster parameters such as powers, delays, and angles, describe the channel at a microscopic level.

With a relatively small frequency separation with respect to the carrier frequency in LTE systems, large and small scale parameters are often assumed to be the same in frequency separated channels $[20,21]$. In [22], simulation results demonstrated that power delay profiles (PDPs) on different frequency bands (with a separation up to $400 \mathrm{MHz}$ ) were highly correlated for both line-of-sight (LOS) and nonLOS scenarios. In [23], experimental results showed that angles of arrival (AoAs) of strong specular signals did not change over the $25 \mathrm{MHz}$ frequency range. Also in [24], it was concluded that power angle spectra (PAS) are approximately identical for the uplink and downlink with a frequency separation of $68 \mathrm{MHz}$. Results showed that both PDP and PAS did not depend on carrier frequency for the LOS and nonLOS scenarios based on extensive measurement campaigns, if the carrier frequency is below $6 \mathrm{GHz}$ [20, 21]. Measurement results in [25] further supported the spatial reciprocity of uplink and downlink channel with a frequency separation up to $200 \mathrm{MHz}$.

2.1.2. Fading Coefficients. It is often assumed that the frequency separation in mobile cellular systems is significantly larger than the coherence bandwidth of the propagation channel, and hence the instantaneous fast fading characteristics of frequency separated channels are uncorrelated. However, in practical frequency-diversity systems, the frequency separation of different channels could be smaller than the coherence bandwidth. For example, the minimum band gap between downlink and uplink can be as low as
$5 \mathrm{MHz}$ in LTE FDD [26]. Carrier aggregation technology has been introduced in LTE-Advanced, where frequency separation between two adjacent subbands (intra- or interband) can be very small [27]. Also, in some frequency-diversity systems, small frequency separation exists due to spectrum and frequency planning constraints [28]. Consequently, fast fading characteristics of frequency separated channels might be correlated, depending on the frequency separation and the channel frequency correlation function (FCF) [29]. In the literature, several techniques have been proposed to generate two or any number of frequency correlated Rayleigh fading sequences [30].

\subsection{Measurement on Frequency Separated Channels. Very} few measurement results were reported in the literature, with a focus on investigating fading correlation of frequency separated channels. In [29], measurement results showed that the correlation decreased rapidly in an example of Rayleigh channel, as the frequency separation increases. As discussed in Section 2.1, fast fading characteristics of frequency separated channels might be correlated, depending on the frequency separation and the propagation channel. This is demonstrated in the following using experimental data.

2.2.1. Measurement Campaign Description. A detailed description of the measurement campaign was given in [31] and hence is only outlined here. The measurements were performed inside a building in a typical microcell non-LOS urban scenario with a wideband MIMO channel sounder [32]. The channel was sampled at $60 \mathrm{~Hz}$, to cope with channel variations due to the movements of the $\mathrm{Rx}$ antennas and other changes in the channel. During the measurement, the $\mathrm{Rx}$ antennas were moved along a $1 \mathrm{~m} \times 1 \mathrm{~m}$ square marked on the floor with $20 \mathrm{~s}$ measurement time. The measurements were performed at $2300 \mathrm{MHz}$ with an effective bandwidth of $30 \mathrm{MHz}$. Note that the $\mathrm{Rx}$ antennas were kept at the same orientation with respect to the environment during the measurement, to ensure that the signals received by the $\mathrm{Rx}$ antennas have similar impinging spatial angles, and hence the recorded channels were stationary. Note that measurement data for two representative cases, selected from a huge pool of available measurements, are presented as examples here.

2.2.2. Analysis and Results. The time-variant transfer function of the measured channel $h_{\text {meas }}(t, f)$ is calculated by taking the Fourier transform of the measured time-variant channel $h_{\text {meas }}(t, \tau)$ in the delay domain. An illustration of the time-variant transfer function of the measured channel is shown in Figure 2. The frequency spacing between subchannels is $0.2667 \mathrm{MHz}$. The measured channel is Rayleigh faded with Rician $K$ factor close to 0 . The fading correlation among the frequency separated subchannels is shown in Figure 3, analyzed with the method suggested in [29]. The correlation generally decreases as the frequency separation between subchannels increases, as expected. The measured correlations are up to 0.8 and 0.6 for a frequency separation of $5 \mathrm{MHz}$ and $10 \mathrm{MHz}$, respectively. 


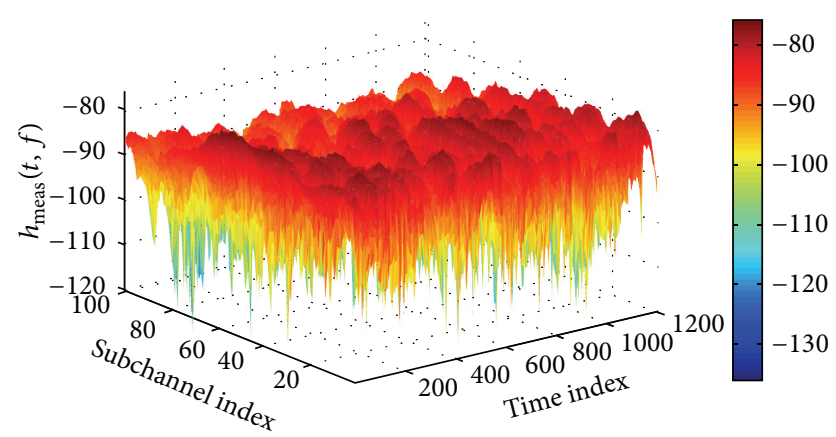

Figure 2: An illustration of one measured channel $h_{\text {meas }}(t, f)$. The channel was sampled at $60 \mathrm{~Hz}$ in the time domain.

The cross-correlation among frequency separated subchannels for a different measurement case is shown in Figure 4. The correlation drops much faster as the frequency separation increases, compared with the results shown in Figure 3. The measured correlations are close to 0.4 and 0 for a frequency separation of $5 \mathrm{MHz}$ and $10 \mathrm{MHz}$, respectively.

As demonstrated by the experimental results, fast fading characteristics of frequency separated channels might be correlated, depending on the frequency separation and the propagation environment.

2.3. Summary of Requirements. Based on the measurement results and the results reported in the literature, it can be summarized that

(i) large and small scale parameters in uplink and downlink channels are highly correlated both for the TDD and FDD systems, and hence large and small scale parameters used in the downlink channels can be adopted for the uplink channels directly;

(ii) fading coefficients for the uplink and downlink are fully correlated in TDD systems, while fading coefficients of downlink and uplink might be correlated in FDD systems, depending on the frequency separation, and the channel FCF.

Realistic uplink channel models should be reciprocal to downlink channel models in TDD systems. More specifically, we have

(1) identical temporal characteristics, for example, Doppler spectrum and temporal correlation.

(2) spatial reciprocity, that is, the same continuous PAS is selected as the target for the uplink and downlink;

(3) fully correlated uplink and downlink fading CIRs experienced by the DUT, that is, $\mathbf{h}_{d}=\mathbf{h}_{u}$. We have

$$
\rho_{T}=\operatorname{corr}\left(\mathbf{h}_{d}, \mathbf{h}_{u}\right)=\frac{\operatorname{cov}\left(\mathbf{h}_{d}, \mathbf{h}_{u}\right)}{\sqrt{\operatorname{cov}\left(\mathbf{h}_{d}, \mathbf{h}_{d}\right) \cdot \operatorname{cov}\left(\mathbf{h}_{u}, \mathbf{h}_{u}\right)}}=1
$$

where $\operatorname{corr}()$ and $\operatorname{cov}()$ are the correlation operator and covariance operator, respectively.
As for the FDD case, the difference is that the fading CIRs in uplink channel might or might not be correlated with those in the downlink channel, depending on the downlink and uplink frequency separation and the channel coherence bandwidth. That is, it would be desirable to create the uplink and downlink fading CIRs with an arbitrary target crosscorrelation $\rho_{F}$ :

$$
\rho_{F}=\operatorname{corr}\left(\mathbf{h}_{d}, \mathbf{h}_{u}\right)
$$

\section{Emulating Bidirectional Channels in MPAC Setups}

In this section, the techniques for emulating bidirectional channels in MPAC setups are presented. First, the technique to emulate downlink channel models is revisited. After that the techniques to emulate uplink channel models are proposed. Since multicluster channel models consist of independently generated single clusters, the discussion in this section is limited to single cluster channel models. The proposed technique can be applied for multicluster channel models, simply by repeating the same procedure for each cluster $[8,33]$.

3.1. Downlink Channel Modeling. The prefaded signal synthesis (PFS) technique, which was proposed in [8], has been widely used for emulating downlink channel models in MPAC setups. As shown in Figure 1, each probe is connected to a channel fading module in the channel emulator, where an arbitrary fading channel can be generated. The objective is to reproduce channels with the desired temporal and spatial characteristics, with the help of the channel emulator and multiple probes.

Assume the number of probes utilized for downlink is $N_{d}$ and the channel impulse response (CIR) for the $n_{d}$ th probe for downlink is denoted by $\sqrt{g_{n_{d}}} \cdot \boldsymbol{\mu}_{n_{d}}$ with $n_{d}=\left[1, \ldots, N_{d}\right]$, where $\left\{g_{n_{d}}\right\} \in \mathbb{R}^{N_{d} \times 1}$ is the power weight vector for the $N_{d}$ downlink probes. $\left\{\boldsymbol{\mu}_{n_{d}}\right\} \in \mathbb{C}^{N_{d} \times N_{i r}}$ are the $N_{d}$ unweighted complex CIRs. $N_{i r}$ is the length of the CIR. Then the CIR $\mathbf{h}_{d} \in \mathbb{C}^{1 \times N_{i r}}$ experienced by a DUT in the downlink, assuming an isotropic antenna pattern, is

$$
\mathbf{h}_{d}=\sum_{n_{d}=1}^{N_{d}} \sqrt{g_{n_{d}}} \cdot \boldsymbol{\mu}_{n_{d}} .
$$

Note that the propagation coefficients from the OTA probes to the test area center are neglected, as they are the same after calibration. The unweighted fading CIR for $n_{d}$ th probe, for a specified Tx antenna and specified polarization, can be simplified according to [8], as follows:

$$
\boldsymbol{\mu}_{n_{d}}(t)=\sum_{m=1}^{M} c_{m} \cdot \exp \left(j 2 \pi v_{m} t+\theta_{n_{d}, m}\right)
$$

where $c_{m}, v_{m}$ denote the complex constant amplitude and the Doppler frequency for the $m$ th subpath of the cluster, respectively. $\theta_{n_{d}, m}$ denotes the random initial phase for the 


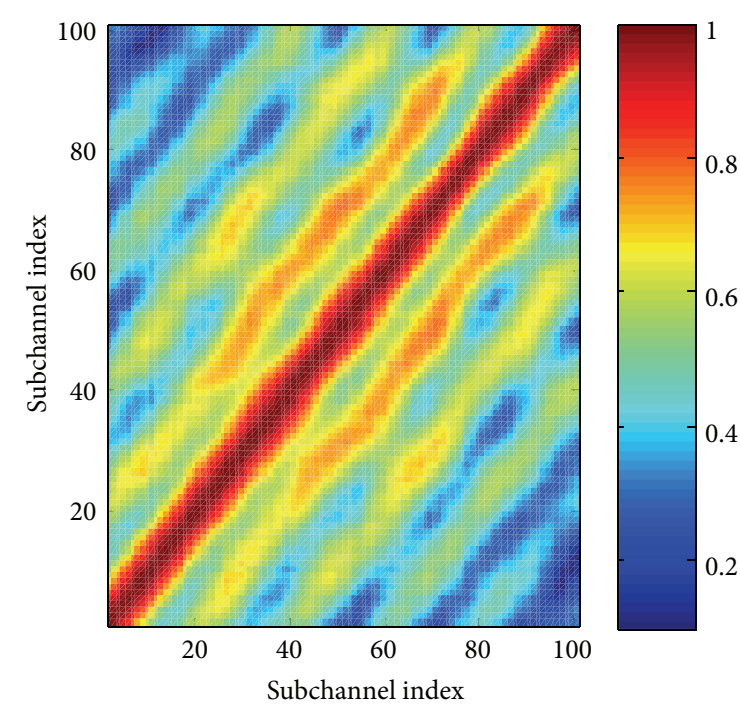

(a)

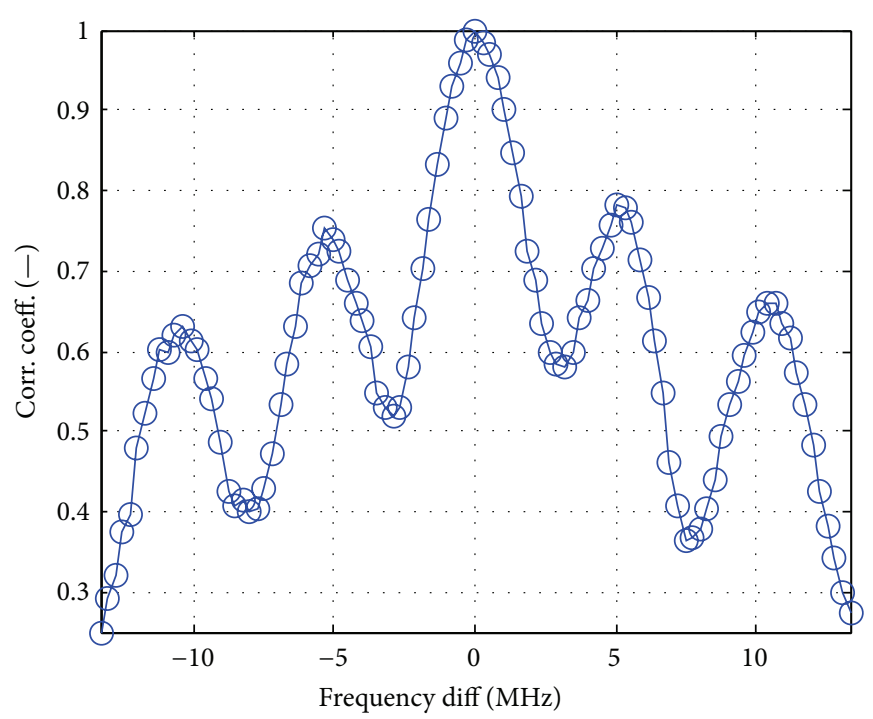

(b)

FIGURE 3: Cross-correlation between subchannels (a) and cross-correlation between the middle subchannel and the other subchannels (b).

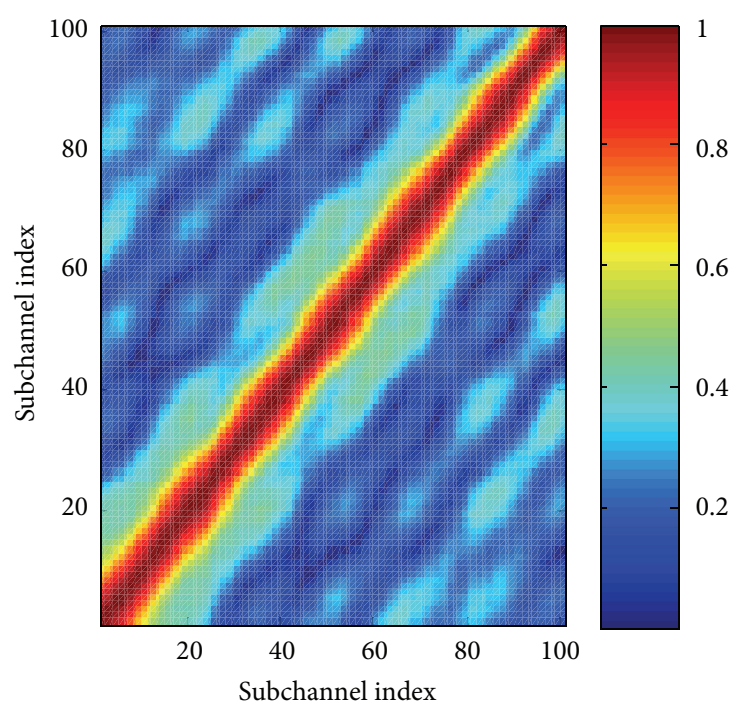

(a)

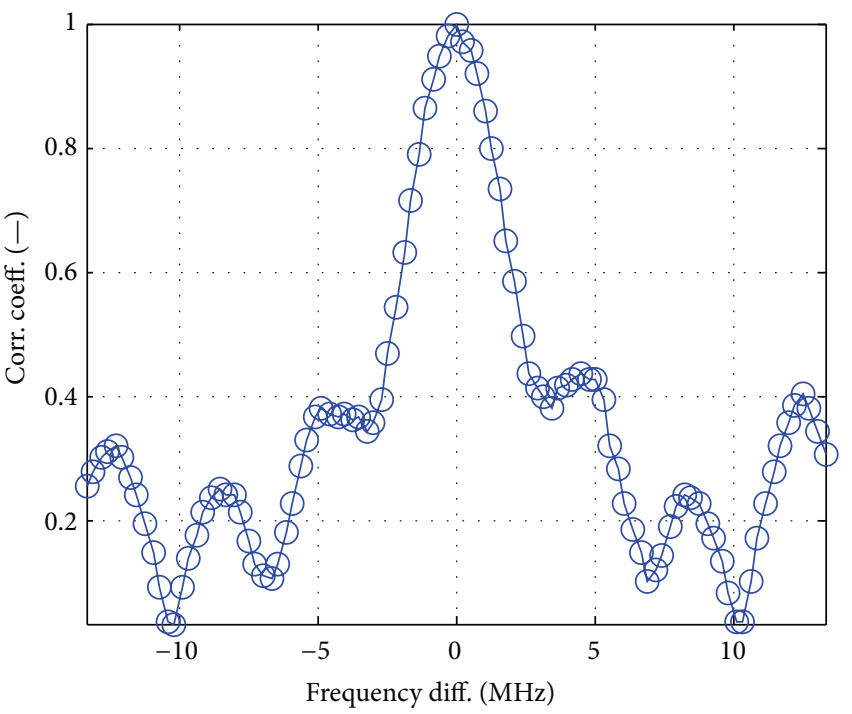

(b)

FIGURE 4: Cross-correlation between subchannels for other measurement data (a) and cross-correlation between middle subchannel and the other subchannels (b).

$m$ th subpath for $n_{d}$ th probe. $m=[1, \ldots, M]$ is the subpath index. The unweighted fading CIRs $\left\{\boldsymbol{\mu}_{n_{d}}\right\}$ transmitted from $N_{d}$ probes belong to Class II stochastic channel simulator as detailed in [34]. That is, each OTA antenna, contributing to the same cluster, transmits independent fading CIRs with identical statistics [8]. As $\mathbf{h}_{d}$ is formed as a linear combination of contributions from multiple probes, it has identical statistics as well.

With the PFS technique, the desired channel temporal characteristics, for example, fading distribution, temporal correlation, and Doppler spectrum, can be reproduced even with a single probe. Multiple probes around the DUT are utilized only to reproduce the spatial characteristics of the channel models at the $\mathrm{Rx}$ side, which are crucial for MIMO capable terminals. To reproduce the channel spatial characteristics in the test zone at the $\mathrm{Rx}$, appropriate power weights $\left\{g_{n_{d}}\right\}$ are allocated to the OTA probes. The goal is to minimize the deviation between the theoretical spatial correlation resulting from the target continuous PAS and the emulated spatial correlation resulting from the discrete PAS, with its shape characterized by the discrete angular positions of the probes and the power weights [33]. The weighted CIRs for a Laplacian shaped spatial cluster with azimuth angle of arrival (AoA) $0^{\circ}$ and azimuth spread (AS) $35^{\circ}$ are shown in Figure 5. To approximate the target continuous PAS, a dominant power weight is allocated to probe 1 (with angular location $0^{\circ}$ ), while a very small (close to 0 ) power weight is allocated to probe 5 (with angular location $180^{\circ}$ ). The discrete PAS follows the target PAS well. The optimization technique to obtain the power weights is detailed in [33] and not repeated here. 


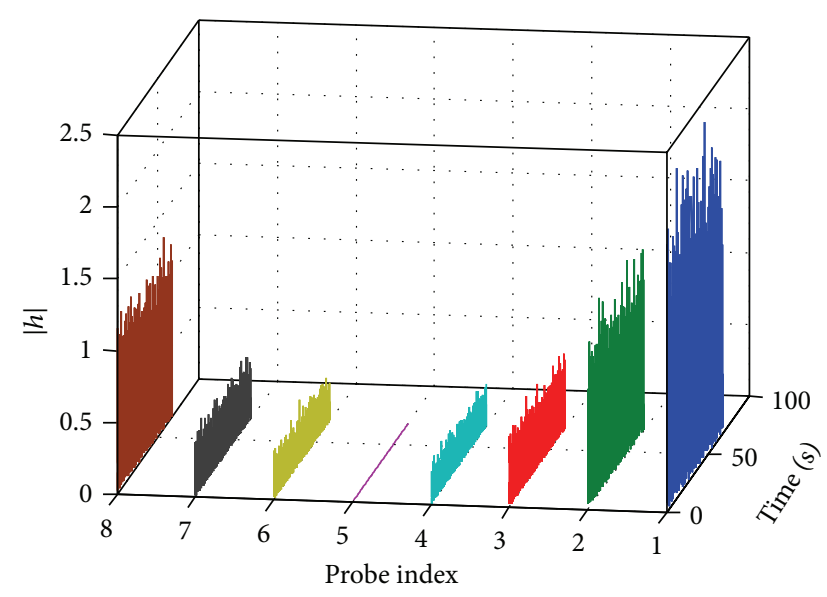

FIGURE 5: An illustration of the weighted CIRs from the eight equally located probes with $N_{d}=8$.

\subsection{Uplink Channel Modeling}

3.2.1. Probe Configuration. An example of the multiprobe anechoic chamber setup that is capable of emulating both uplink and downlink channels is shown in Figure 6. The goal here is to emulate the realistic uplink channels experienced by the signals transmitted from the DUT, with the help of $N_{u}$ probes and the channel emulator in the uplink. A mapping of the uplink channel to each of the uplink probes is, therefore, required for adding realistic uplink fading.

In this paper, symmetric probe configuration indicates that the same set of probes are used in the uplink and downlink, that is, colocated probes for the uplink and downlink. With a symmetric probe configuration, one practical constraint is that the circulators to separate downlink and uplink signals (in Figure 6) are quite limited in frequency range. Moreover, circulators are practically limited in isolating the uplink and downlink (e.g., with an isolation loss of $20 \mathrm{~dB}$ ).

An alternative is to use different (spatially separated) probes to separate the downlink and uplink. Asymmetric probe configurations, where different number of probes or spatially separated probes are adopted for the uplink and downlink, might be more popular in practical setups. The test zone is a geometrical volume inside which the DUT is located and it is required that the target channel models are reproduced accurately inside the test zone. It has been demonstrated that the test area size depends directly on the number of probes [33]. There might be fewer uplink probes by design if a subscriber terminal has fewer transmitting antennas than receiving antennas and the smaller transmitting antenna array size requires fewer uplink probes for the same accuracy of OTA testing. Furthermore, downlink probe configuration has been specified in standardization (e.g., 3GPP), while uplink probe configuration has not been defined.

3.2.2. Objective. Assume that we have $N_{u}$ probes for the uplink and the CIR for the $n_{u}$ th probe is denoted by $\sqrt{w_{n_{u}}} \cdot \boldsymbol{\nu}_{n_{u}}$ with $n_{u}=\left[1, \ldots, N_{u}\right]$, where $\left\{w_{n_{u}}\right\} \in \mathbb{R}^{N_{u} \times 1}$ is the power weight vector for $N_{u}$ uplink probes. $\left\{\boldsymbol{\nu}_{n_{u}}\right\} \in \mathbb{C}^{N_{u} \times N_{i r}}$ are
$N_{u}$ unweighted complex CIRs. Then the CIRs $\mathbf{h}_{u} \in \mathbb{C}^{1 \times N_{i r}}$ experienced by the DUT in the uplink, assuming isotropic antenna pattern and neglecting propagation coefficients, are

$$
\mathbf{h}_{u}=\sum_{n_{u}=1}^{N_{u}} \sqrt{w_{n_{u}}} \cdot \boldsymbol{v}_{n_{u}} .
$$

In the following sections, techniques to emulate the uplink channel models for different probe configurations are detailed for both TDD and FDD systems. The goal is to find appropriate fading CIRs $\left\{\boldsymbol{\nu}_{n_{u}}\right\}$ and power weights $\left\{w_{n_{u}}\right\}$ for the uplink, so that the requirements discussed in Section 3.2.2 are fulfilled for FDD and TDD systems.

\subsubsection{Symmetric Probe Configurations}

(a) TDD Systems. Channel reciprocity for TDD systems in symmetric probe configurations can be achieved simply by reusing the downlink power weights and fading CIRs in the uplink, that is, $w_{n}=g_{n}$ and $\boldsymbol{\mu}_{n}=\boldsymbol{\nu}_{n}$ with $n=[1, \ldots, N]$.

(b) FDD Systems. As explained earlier, in typical systems the frequency separation is high with respect to the channel coherence bandwidth, and it is a valid assumption that the uplink and downlink fading are independent and channel realizations should be uncorrelated. Anyhow there might be cases, where frequency separation is small and/or the FCF decreases very slowly, resulting in correlated uplink and downlink channels.

The procedure to create correlated uplink and downlink CIRs for symmetric probe configurations is detailed below.

Firstly, two sets of unweighted CIRs $\left\{\xi_{n}^{1}\right\} \in \mathbb{C}^{N \times N_{i r}}$ and $\left\{\boldsymbol{\xi}_{n}^{2}\right\} \in \mathbb{C}^{N \times N_{i r}}$, each CIR with independent and identical distribution, are generated according to (4). Note that the created CIRs are with identical temporal characteristics.

Secondly, the CIRs for the downlink $\left\{\boldsymbol{\mu}_{n}\right\}$ and uplink $\left\{\boldsymbol{\nu}_{n}\right\}$ with desired correlation $\rho_{F}$ can be created by multiplying the uncorrelated fading CIRs $\left\{\boldsymbol{\xi}_{n}^{1}\right\}$ and $\left\{\boldsymbol{\xi}_{n}^{2}\right\}$ with Cholesky factorization of the correlation matrix, according to [35], as

$$
\left[\begin{array}{l}
\boldsymbol{\mu}_{n}(t) \\
\nu_{n}(t)
\end{array}\right]=M\left[\begin{array}{l}
\xi_{n}^{1}(t) \\
\xi_{n}^{2}(t)
\end{array}\right], \text { for } n=[1, \ldots, N],
$$

where $M=\operatorname{chol}\left(\left[\begin{array}{cc}1 & \rho_{F}^{H} \\ \rho_{F} & 1\end{array}\right]\right)$ with $(\cdot)^{H}$ being the complex conjugate operator and $t=\left[1, \ldots, N_{i r}\right] \cdot \operatorname{chol}(\cdot)$ is the Cholesky factorization operator [35].

As the probes are colocated, the spatial reciprocity can be achieved simply by setting $w_{n}=g_{n}$ with $n=[1, \ldots, N]$. The generated fading CIRs for the uplink $\left\{\boldsymbol{\nu}_{n}\right\}$ are with desirable temporal characteristics and correlated with the downlink CIRs with a desired correlation value $\rho_{F}$.

3.2.4. Asymmetric Probe Configurations. The methods proposed in Section 3.2.3, however, only work for the cases when the uplink and downlink probes are colocated (i.e., symmetric probe configurations). In this section, a novel technique is proposed for asymmetric probe configurations. In the following, $N_{u}<N_{d}$ is assumed for notational 


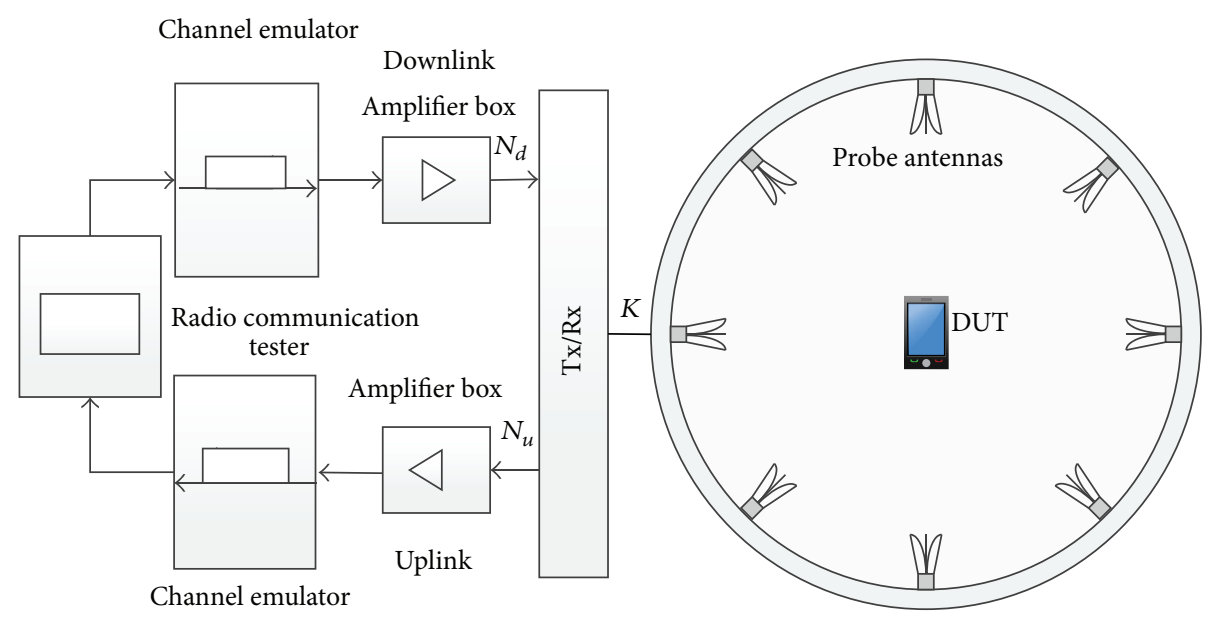

FiguRE 6: An example of a symmetric MPAC setup that supports emulating both uplink and downlink channels. $N_{d}$ and $N_{u}$ probes are used for the downlink and uplink, respectively, with $N_{u} \leqq N_{d} \leqq K$. Note that the same probe can be used for transmitting in the downlink and receiving in the uplink with the help of a circulator.

simplicity, although the proposed technique is not limited to this assumption.

As explained in Section 3.2.2, to obtain the spatial reciprocity, the same target PAS should be used when calculating the power weights in the uplink and downlink. The optimization techniques to obtain probe power weights for a specific probe configuration and target PAS were detailed in [33]. An illustration of the power weights for the downlink and uplink channel model for an asymmetric probe configuration is shown in Figure 7. It is expected that the emulation accuracy in terms of spatial correlation in the uplink is worse, as few probes are utilized. However, the required test area size for the uplink is also smaller, as fewer Tx antennas than Rx antennas at the MS side are expected.

The procedure to generate correlated uplink and downlink CIRs with the same temporal characteristics for asymmetric probe configurations is detailed as follows.

(1) Divide $N_{d}$ downlink fading CIRs $\left\{\boldsymbol{\mu}_{n_{d}}\right\}$ into $N_{u}$ subgroups so that each uplink CIR has an associated subset of downlink CIRs. Assume that the number of downlink fading CIRs in $n_{u}$ th subgroup is $I_{n_{u}}$, with $I_{n_{u}} \geq 1$ and $\sum_{n_{u}=1}^{N_{u}} I_{n_{u}}=N_{d}$. Note that there is no restriction on how $N_{d}$ downlink CIRs $\left\{\boldsymbol{\mu}_{n_{d}}\right\}$ are divided into $N_{u}$ subgroups. $i$ th $\left(i \in\left[1, I_{n_{u}}\right]\right)$ CIR in $n_{u}$ th subgroup corresponds to the $\left(i+\sum_{l=1}^{n_{u}} I_{l-1}\right)$ downlink CIR with $I_{0}=0$ in $N_{d}$ downlink CIRs.

(2) Generate a set of unweighted CIRs $\left\{\boldsymbol{\varsigma}_{n_{u}}\right\} \in \mathbb{C}^{N_{u} \times N_{i r}}$ with independent and identical distributions, according to (4).

(3) Obtain uplink CIRs $\left\{\boldsymbol{v}_{n_{u}}\right\}$ by expressing the uplink CIR $v_{n_{u}}$ in terms of the downlink CIRs belonging to $n_{u}$ th subgroup and $n_{u}$ th unweighted CIR $\boldsymbol{\varsigma}_{n_{u}}$. This involves expressing $v_{n_{u}}$ in the form

$$
\boldsymbol{\nu}_{n_{u}}=a \cdot \boldsymbol{\varsigma}_{n_{u}}+b \cdot \sum_{i=1}^{I_{n_{u}}} \boldsymbol{\mu}_{i+\sum_{l=1}^{n_{u}} I_{l-1}},
$$

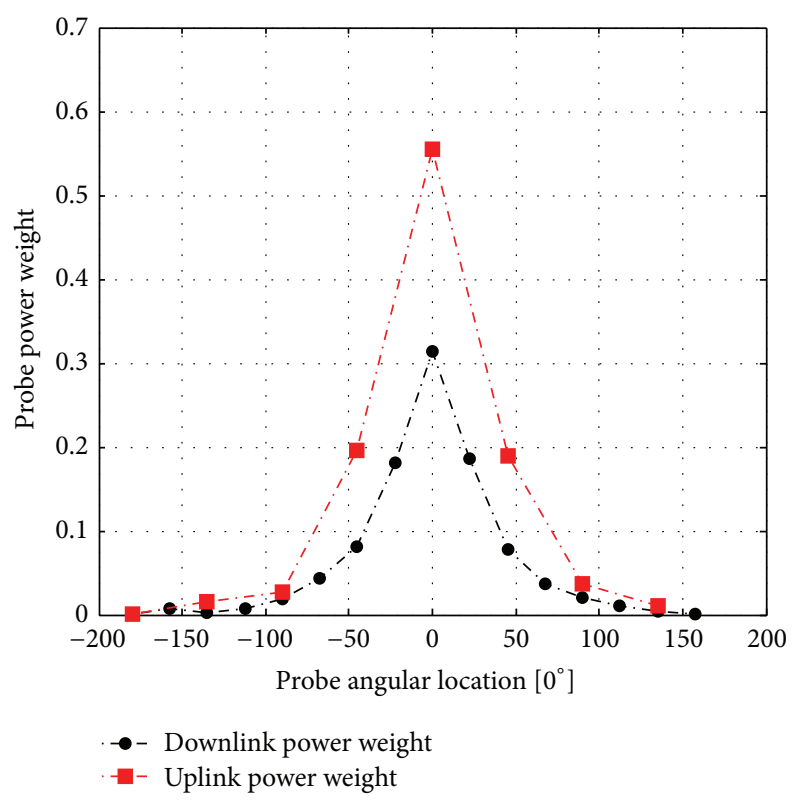

Figure 7: Power weights for the downlink (with $N_{d}=16$ ) and uplink (with $N_{u}=8$ ) channel model. The target PAS for the uplink and downlink is a single Laplacian shaped spatial cluster with AoA $=0^{\circ}$ and $\mathrm{AS}=35^{\circ}$.

where $a$ and $b$ are the complex coefficients to be calculated. $n_{u}$ th uplink CIR $\nu_{n_{u}}$ may then be defined in terms of the downlink CIRs belonging to $n_{u}$ th subgroup and $n_{u}$ th complex CIR $\boldsymbol{\varsigma}_{n_{u}}$. The objective is to obtain $a$ and $b$, such that (2) is fulfilled. As explained earlier, $w_{n_{u}}$ can be obtained based on the uplink probe configuration and the same target PAS used for the downlink channel. The target correlation $\rho_{F}$ between the uplink and downlink fading CIRs can be calculated by methods known in the art. An example is through the FCF of radio channels, which 
can be obtained via Fourier transform of the channel average PDP [29]. As indicated above, $\left|\rho_{F}\right|=1$ for TDD systems and a predefined $\left|\rho_{F}\right|<1$ for FDD systems are targeted.

(4) Based on the properties of $\left\{\boldsymbol{s}_{n_{u}}\right\}$ and $\left\{\boldsymbol{\mu}_{n_{d}}\right\}$, we have

$$
\begin{gathered}
a=\frac{\left(1-\widehat{c}_{n_{u}}\right)}{\sqrt{\left|1-\widehat{c}_{n_{u}}\right|^{2}+I_{n_{u}} \cdot\left|\widehat{c}_{n_{u}}\right|^{2}}}, \\
b=\frac{\widehat{c}_{n_{u}}}{\sqrt{\left|1-\widehat{c}_{n_{u}}\right|^{2}+I_{n_{u}} \cdot\left|\widehat{c}_{n_{u}}\right|^{2}}},
\end{gathered}
$$

and $\widehat{c}_{n_{u}}$ is calculated by solving the equation,

$$
c_{n_{u}}=\frac{\widehat{c}_{n_{u}}}{\sqrt{\left|1-\widehat{c}_{n_{u}}\right|^{2}+I_{n_{u}} \cdot\left|\widehat{c}_{n_{u}}\right|^{2}}}
$$

where $\left\{c_{n_{u}}\right\}$ is a set of coefficients that satisfy $\sum_{n_{u}=1}^{N_{u}} c_{n_{u}}$. $\sqrt{w_{n_{u}}} \cdot \sum_{i=1}^{I_{n_{u}}} \sqrt{g_{i+\sum_{l=1}^{n_{u}} I_{l-1}}}=\rho_{F}$. Note that we have $N_{u}$ coefficients $\left\{c_{n_{u}}\right\}$ to solve with only one equation. Any set of $\left\{c_{n_{u}}\right\}$ satisfying the above equation can be used.

It can be easily checked that the fading correlation between uplink channel $\mathbf{h}_{u}$ and downlink channel $\mathbf{h}_{d}$ can reach the target correlation $\rho_{F}$. A simple illustration of the procedure to generate the desired uplink CIRs (the downlink and uplink probes are selected to be $N_{d}=3$ and $N_{u}=2$, resp.) with an example case is shown in the following.

Split downlink Irs into two subsets:

$$
\left.\begin{array}{c}
\boldsymbol{\mu}_{1} \\
\boldsymbol{\mu}_{2} \\
\boldsymbol{\mu}_{3}
\end{array}\right\}
$$

Select the appropriate coefficients $c_{n_{u}}$ :

$$
c_{1} \cdot \sqrt{\omega_{1}} \sqrt{g_{1}}+c_{2} \cdot \sqrt{\omega_{2}}\left(\sqrt{g_{2}}+\sqrt{g_{3}}\right)=\rho .
$$

Calculate the appropriate coefficients $\widehat{c}_{n_{u}}$ :

$$
\begin{aligned}
& c_{1}=\frac{\widehat{c}_{1}}{\sqrt{\left|1-\widehat{c}_{1}\right|^{2}+\left|\widehat{c}_{1}\right|^{2}}}, \\
& c_{2}=\frac{\widehat{c}_{2}}{\sqrt{\left|1-\widehat{c}_{2}\right|^{2}+2\left|\widehat{c}_{2}\right|^{2}}} .
\end{aligned}
$$

Obtain uplink Irs: $\nu_{1}=\frac{\left(1-\widehat{c}_{1}\right) \cdot \nu_{1}+\widehat{c}_{1} \cdot \mu_{1}}{\sqrt{\left|1-\widehat{c}_{1}\right|^{2}+\left|\widehat{c}_{1}\right|^{2}}}$,

$\nu_{2}=\frac{\left(1-\widehat{c}_{2}\right) \cdot \nu_{1}+\widehat{c}_{2} \cdot\left(\mu_{2}+\mu_{3}\right)}{\sqrt{\left|1-\widehat{c}_{2}\right|^{2}+2\left|\widehat{c}_{2}\right|^{2}}}$,

$\operatorname{cov}\left(\boldsymbol{\nu}_{1}, \boldsymbol{\mu}_{1}\right)=c_{1}$,

$\operatorname{cov}\left(\boldsymbol{\nu}_{1}, \boldsymbol{\mu}_{2}\right)=0$,

$\operatorname{cov}\left(\boldsymbol{\nu}_{1}, \boldsymbol{\mu}_{3}\right)=0$,

$\operatorname{cov}\left(\boldsymbol{\nu}_{2}, \boldsymbol{\mu}_{1}\right)=0$,

$\operatorname{cov}\left(\boldsymbol{\nu}_{2}, \boldsymbol{\mu}_{2}\right)=c_{2}$,

$\operatorname{cov}\left(\nu_{2}, \mu_{3}\right)=c_{2}$,

$\operatorname{corr}\left(\mathbf{h}_{d}, \mathbf{h}_{u}\right)=\operatorname{corr}\left(\sum_{n_{d}} \sqrt{g_{n_{d}}} \cdot \boldsymbol{\mu}_{n_{d}}, \sum_{n_{u}} \sqrt{\omega_{n_{u}}} \cdot \boldsymbol{\nu}_{n_{u}}\right)$

$=\frac{\operatorname{cov}\left(\sqrt{g_{1}} \cdot \boldsymbol{\mu}_{1}+\sqrt{g_{2}} \cdot \boldsymbol{\mu}_{2}+\sqrt{g_{3}} \cdot \boldsymbol{\mu}_{3}, \sqrt{\omega_{1}} \cdot \boldsymbol{\nu}_{1}+\sqrt{\omega_{2}} \cdot \boldsymbol{\nu}_{2}\right)}{\sqrt{\operatorname{cov}\left(\sqrt{g_{1}} \cdot \boldsymbol{\mu}_{1}+\sqrt{g_{2}} \cdot \boldsymbol{\mu}_{2}+\sqrt{g_{3}} \cdot \boldsymbol{\mu}_{3}, \sqrt{g_{1}} \cdot \boldsymbol{\mu}_{1}+\sqrt{g_{2}} \cdot \boldsymbol{\mu}_{2}+\sqrt{g_{3}} \cdot \boldsymbol{\mu}_{3}\right) \cdot \operatorname{cov}\left(\sqrt{\omega_{1}} \cdot \boldsymbol{\nu}_{1}+\sqrt{\omega_{2}} \cdot \boldsymbol{\nu}_{2}, \sqrt{\omega_{1}} \cdot \boldsymbol{\nu}_{1}+\sqrt{\omega_{2}} \cdot \boldsymbol{\nu}_{2}\right)}}$

$=\frac{\sqrt{g_{1}} \cdot \sqrt{\omega_{1}} \cdot c_{1}+\sqrt{g_{2}} \cdot \sqrt{\omega_{2}} \cdot c_{2}+\sqrt{g_{3}} \cdot \sqrt{\omega_{2}} \cdot c_{2}}{\sqrt{\left(g_{1}+g_{2}+g_{3}\right) \cdot\left(\omega_{1}+\omega_{2}\right)}}=\sqrt{g_{1}} \cdot \sqrt{\omega_{1}} \cdot c_{1}+\sqrt{g_{2}} \cdot \sqrt{\omega_{2}} \cdot c_{2}+\sqrt{g_{3}} \cdot \sqrt{\omega_{2}} \cdot c_{2}=\rho$. 
TABLE 1: Details of the three representative channels.

\begin{tabular}{lcccc}
\hline Scenario & Target channel model & $N_{d}$ & $N_{u}$ & $I_{n_{u}}$ for $n_{u} \in\left[1, N_{u}\right]$ \\
\hline A & A uniform PAS & 16 & 4 & 4 \\
B & A single Laplacian shaped spatial cluster with AoA $=0^{\circ}$ and AS $=35^{\circ}$ & 16 & 8 & 2 \\
C & A single Laplacian shaped spatial cluster with AoA $=0^{\circ}$ and AS $=35^{\circ}$ & 16 & 4 & 4 \\
\hline
\end{tabular}

3.2.5. Discussions. The maximal achievable correlation between the uplink and downlink depends on the coefficients $\left\{c_{n_{u}}\right\}$, uplink power weights $\left\{w_{n_{u}}\right\}$, and downlink power weights $\left\{g_{n_{d}}\right\}$ and how the downlink CIRs $\left\{\boldsymbol{\mu}_{n_{u}}\right\}$ are divided into $N_{u}$ subgroups. From (9), we have $\left|c_{n_{u}}\right| \leqq 1 / \sqrt{I_{n_{u}}}$, and hence the maximal achievable fading correlation is

$$
\rho_{\max }=\sum_{n_{u}=1}^{N_{u}} \frac{1}{\sqrt{I_{n_{u}}}} \cdot \sqrt{w_{n_{u}}} \cdot \sum_{i=1}^{I_{n_{u}}} \sqrt{g_{i+\sum_{m=1}^{n_{u}} I_{m-1}}} .
$$

Note that symmetric probe configurations are a special class of assymetric case, where we have $n_{d}=n_{u}=n, N_{u}=$ $N_{d}=N, w_{n_{u}}=g_{n_{d}}$, and $I_{n_{u}}=1$ for $n_{u}=\left[1, \ldots, N_{u}\right] \cdot \rho_{\max }=$ 1 is reached when $c_{n}=1$ is selected. That is, the same set of CIRs for the downlink is reused for the uplink. Arbitrary correlation can be achieved by selecting appropriate values for $\left\{c_{n}\right\}$. For example, uplink and downlink fading CIRs can be uncorrelated by setting $c_{n}=0$ for $n=[1, \ldots, N]$.

From (14), we can conclude that, for an asymmetric probe configuration, $\rho_{\max }=1$ can only be achieved when the PAS is uniform (i.e., $g_{n_{d}}=1 / N_{d}$ for $n_{d}=\left[1, \ldots, N_{d}\right]$ and $w_{n_{u}}=$ $1 / N_{u}$ for $n_{u}=\left[1, \ldots, N_{u}\right]$ ), subgroups are equal (i.e., $I_{n_{u}}=$ $N_{u} / N_{d}$ for $\left.n_{u}=\left[1, \ldots, N_{u}\right]\right)$, and $c_{n_{u}}=1 / \sqrt{I_{n_{u}}}$ is set. For nonuniform PAS cases, the achievable correlation is upbound according to (14) and will be slightly smaller than 1 .

\section{Simulation Results}

Three representative scenarios are selected in the simulations to demonstrate the proposed technique, as detailed in Table 1. Note that downlink CIRs are evenly divided into subgroups in all three scenarios.

4.1. Spatial Characteristics Reciprocity. As explained earlier, to ensure spatial reciprocity between uplink and downlink channels, the same target continuous PAS is selected for the downlink and uplink. The emulation accuracy in terms of spatial correlation depends directly on number of probes, as explained in $[33,36]$. The spatial correlation is used as a measure to determine how well the spatial characteristic is reproduced. The spatial correlation emulation accuracy for the downlink and uplink for the scenario A is shown in Figure 8. The target curve follows zeroth-order Bessel function of the first kind, as the target PAS is uniform. With this asymmetric probe configuration, the maximal antenna separation in transmitting array on the MS is less than $0.5 \lambda$ for the uplink, while the antenna separation in the receiving array on the MS can be as large as $1.5 \lambda$. Similar conclusions

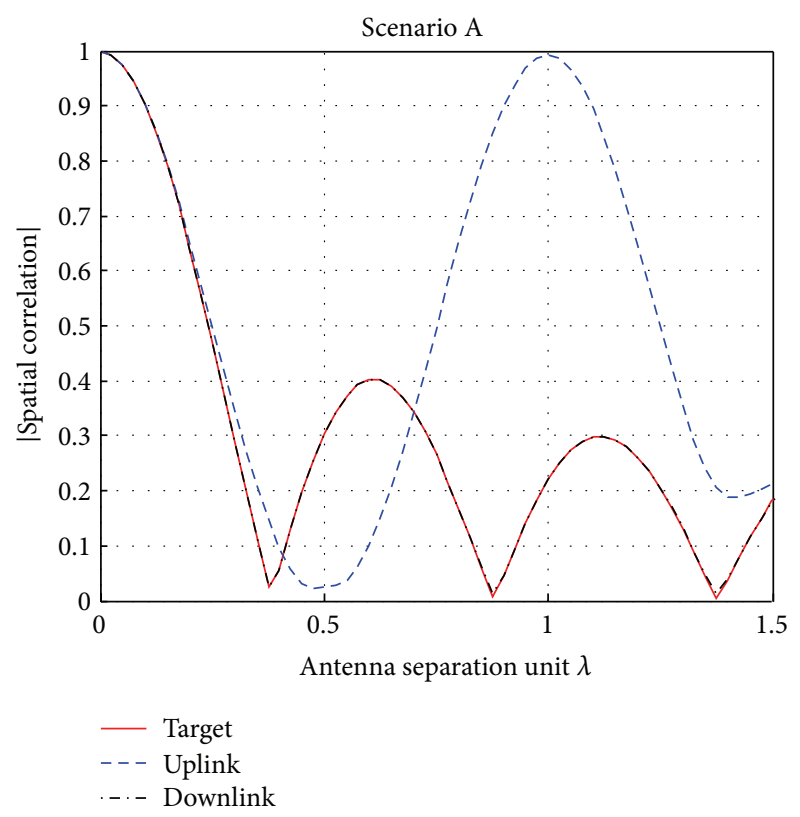

FIGURE 8: Spatial correlation of the downlink (with $N_{d}=16$ ) and uplink (with $N_{u}=4$ ) channel model for scenario A.

can be drawn for the other two scenarios. The discrete PAS for the scenario B is shown in Figure 7.

4.2. Temporal Fading Characteristics Reciprocity. As explained earlier, $\mathbf{h}_{d}$ is formed as a linear combination of contributions from multiple probes, and hence it has identical fading statistics to that of the CIRs from individual probes. As shown in Figure 9, the temporal autocorrelation of the generated downlink CIR $\mathbf{h}_{d}$ and the unweighted fading CIR from 1st probe $\boldsymbol{\mu}_{1}$ match very well. The same results applies for the uplink CIR $\mathbf{h}_{u}$ and $\left\{\boldsymbol{v}_{n}\right\}$, as shown in Figure 9. As both $\left\{\boldsymbol{\nu}_{n_{u}}\right\}$ and $\left\{\boldsymbol{\mu}_{n_{d}}\right\}$ are generated according to (4), the temporal autocorrelation of $\mathbf{h}_{d}$ matches that of $\mathbf{h}_{u}$ well. The same conclusions can be drawn for the other two scenarios. As a conclusion, the temporal fading characteristic reciprocity in the uplink and downlink can be achieved with the proposed technique.

4.3. Fading Correlation between the Downlink and Uplink Channels. To achieve the maximal correlation between the downlink and uplink fading channel, $\left|c_{n_{u}}\right|=1 / \sqrt{I_{n_{u}}}$ for $n_{u} \in\left[1, N_{u}\right]$ is selected for three representative scenarios (i.e, 0.5 for scenario $\mathrm{A}, 1 / \sqrt{2}$ for scenario $\mathrm{B}$, and 0.5 for scenario C). The achieved correlations between the downlink and 


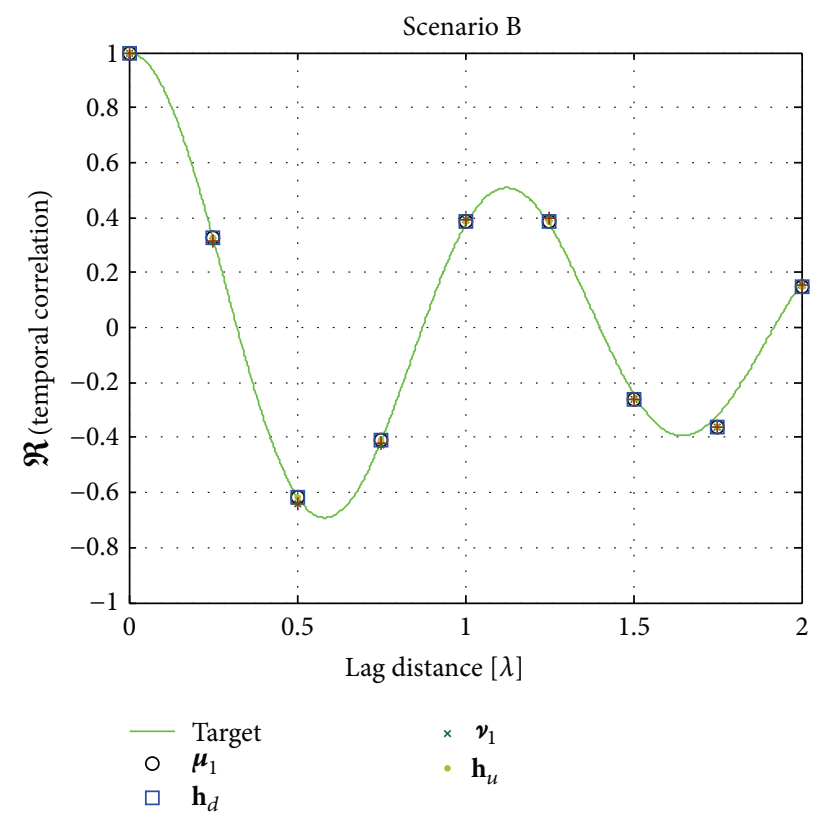

FIGURE 9: Temporal autocorrelations of the target channel model, unweighted fading CIR from 1st probe in the downlink $\boldsymbol{\mu}_{1}$, downlink fading CIR $\mathbf{h}_{d}$, unweighted fading CIR from 1st probe in the uplink $\boldsymbol{\nu}_{1}$, and uplink fading CIR $\mathbf{h}_{u}$ for scenario B. Note that the direction of travel is $30^{\circ}$ in the simulation and the fading CIRs are four samples per wavelength.

uplink fading CIRs $\mathbf{h}_{d}$ and $\mathbf{h}_{u}$, calculated based on correlation definition, are $1,0.97$, and 0.89 for scenarios $\mathrm{A}, \mathrm{B}$, and $\mathrm{C}$, respectively. The values match perfectly the maximal achievable correlation specified in (14). As we can see, though $|\rho|=1$ cannot be reached for nonuniform PAS channel models (e.g., scenario B and scenario C) in asymmetric probe configurations, the achieved correlations are very high (close to 1$)$.

Cross-correlation $\rho$ among the fading CIRs $\left\{\boldsymbol{\mu}_{n_{u}}\right\}$ in the downlink and $\left\{\boldsymbol{\nu}_{n_{u}}\right\}$ in the uplink for the scenario B is shown in Figure 10. We can see that

(1) correlation between the $n_{u}$ th CIR in the uplink $\boldsymbol{v}_{n_{u}}$ and the downlink CIRs in $n_{u}$ th subset is $c_{n_{u}}$, which is $1 / \sqrt{2}$ for the scenario B;

(2) correlation between $\boldsymbol{v}_{n_{u}}$ and the downlink CIRs that are not in $n_{u}$ th subset is 0 ;

(3) correlation among uplink CIRs $\left\{\boldsymbol{v}_{n_{u}}\right\}$ is 0 ;

(4) correlation among downlink CIRs $\left\{\boldsymbol{\mu}_{n_{u}}\right\}$ is 0 .

The results are expected from the equations shown in Section 3.2.4.

Note that the generated uplink CIR can be made correlated with the downlink CIR with an arbitrary value with its magnitude ranging from 0 to the maximal achievable limit, for example, 0.89 for scenario $\mathrm{C}$.

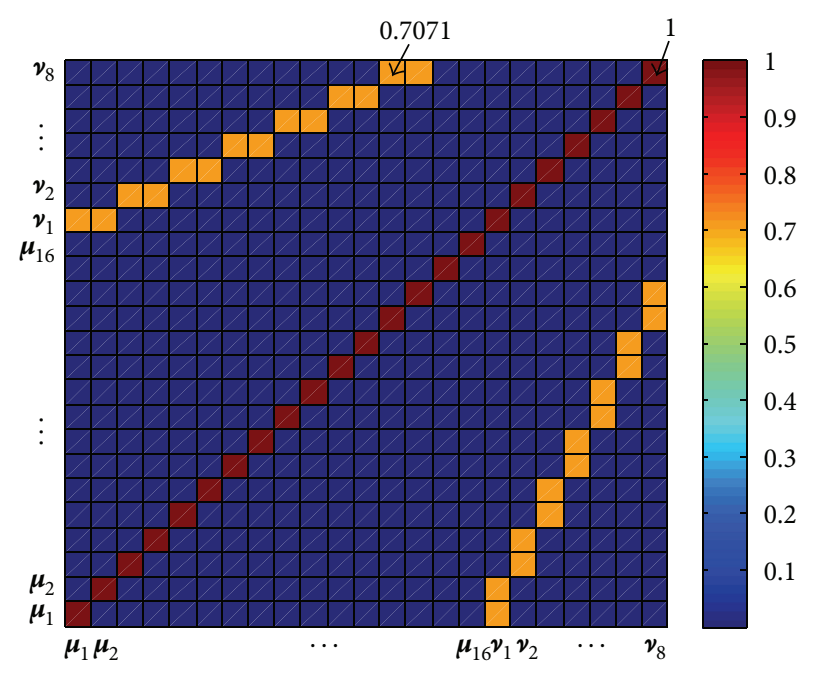

FIgURE 10: Cross-correlation among the fading CIRs $\left\{\boldsymbol{\mu}_{n_{u}}\right\}$ in the downlink and $\left\{\boldsymbol{v}_{n_{u}}\right\}$ in the uplink for the scenario B. The value in the correlation matrix denotes the correlation between two fading CIRs indicated in the $x$-and $y$-axes.

\section{Measurement Validation in the Anechoic Chamber}

A measurement campaign was carried out in a practical setup at Anite Telecoms Oy, Finland, to verify the algorithms presented in Section 3. Figure 11 illustrates the test setup. Eight uniformly located probes on the azimuth ring were connected to an Anite Propsim F32 to synthesize the downlink channel, while four probes were connected to the channel emulator to generate the uplink channel. Note that a channel emulator that is capable of separating uplink and downlink signal (i.e., bidirectional fading modes) is required. Phase and amplitude calibrations are performed for each probe before the measurements with a calibration antenna. The measured spatial correlation and temporal correlation of downlink channels have been well investigated in the literature [3] and not detailed here. The focus of the measurement section is to investigate whether the target fading correlation can be achieved between the downlink and uplink channel. The basic idea is to record the channel impulse responses using the calibration dipole and spectrum analyzer (SA) in the downlink and uplink separately and then calculate the measured fading correlation.

A uniform PAS is selected in the validation measurements for the sake of simplicity. Both TDD and FDD systems with target fading correlation $\rho_{F}=1$ were considered in the measurement campaign. The measured fading correlations match the target values quite well, 0.99 and 1 for the TDD system and FDD system, respectively. The deviation in the TDD system might be due to the system uncertainties in the measurement system. The measured fading profiles for the uplink and downlink for the FDD system are shown in Figure 12. Note that an arbitrary fading correlation ranging from 0 to 1 can be achieved for the FDD system in this case, as explained earlier. 


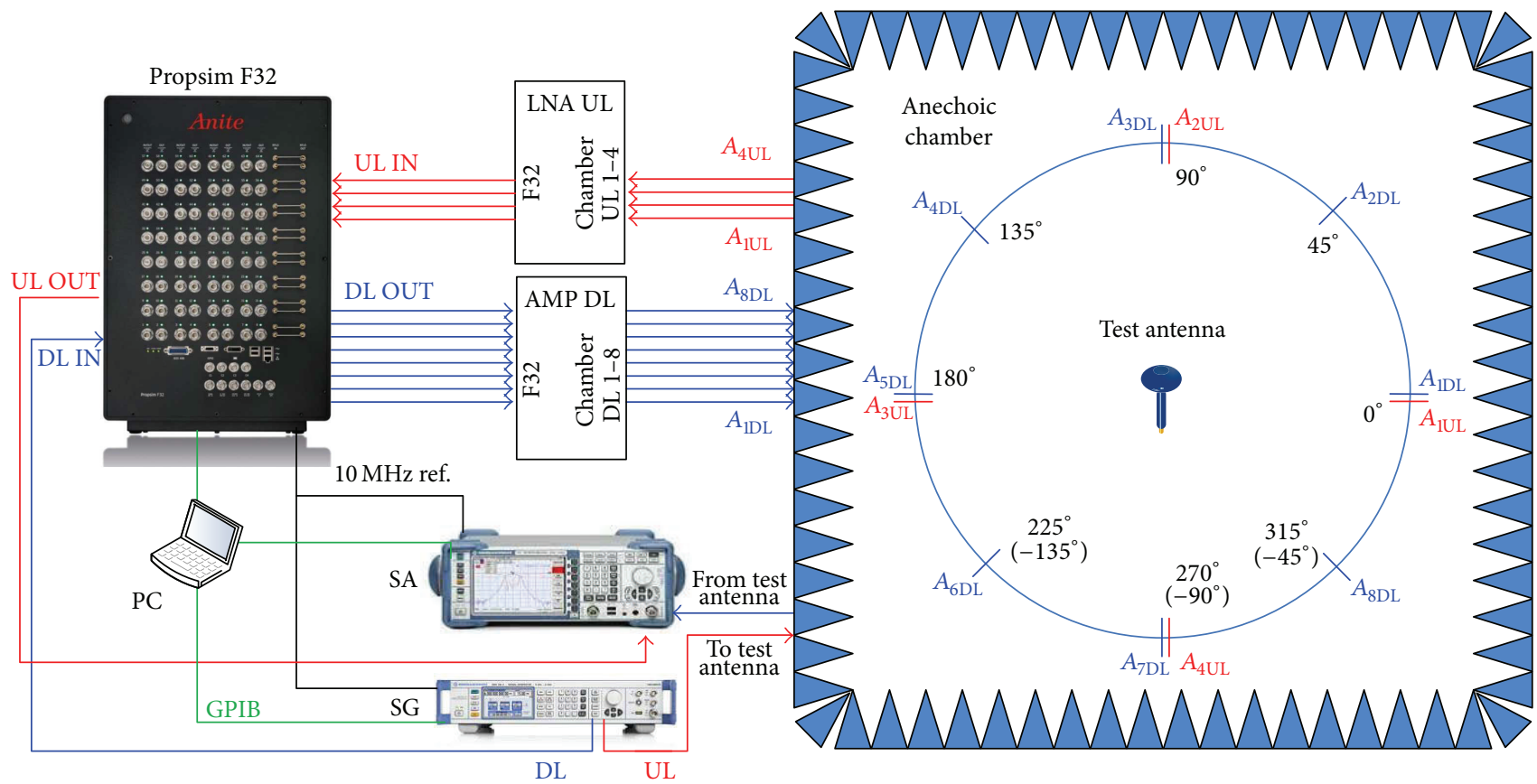

FIGURE 11: An illustration of the measurement system. Blue indicates the downlink and red denotes the uplink. Note that low noise amplifiers (LNAs) were used in the uplink, because the signals received by probes may have low signal to noise ratio (SNR). In the downlink, the amplification is typically done for signals with good SNRs. Different local oscillators are used in the RF channels serving uplink and downlink, especially in FDD case. All local oscillators in a fading emulator (or a set of fading emulators) typically share a common reference clock to guarantee stable synchronization. The reference clock can be taken, for example, from a satellite clock signal and distributed to different units.

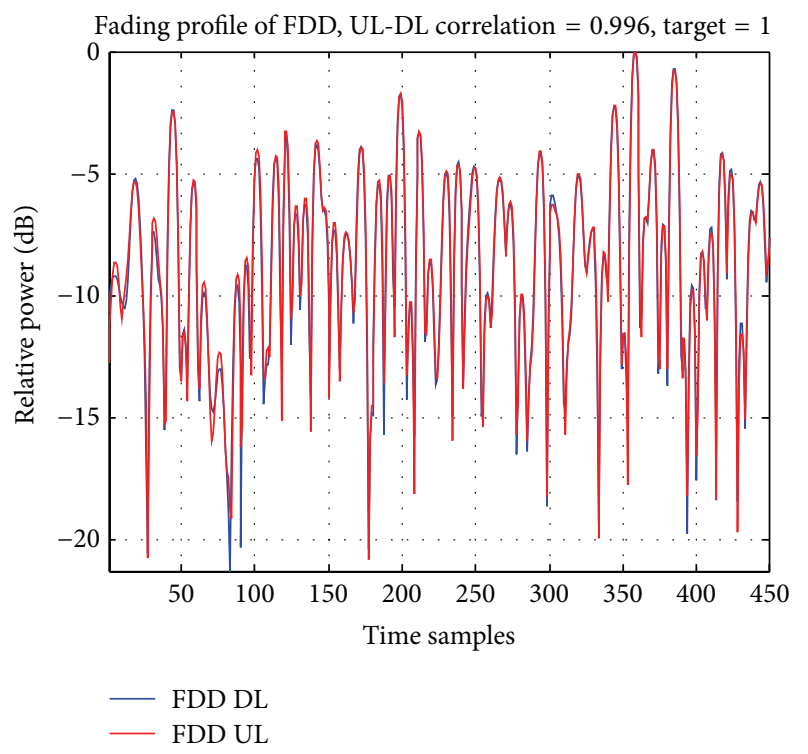

Figure 12: Measured fading profiles for the uplink and downlink for the FDD system. DL and UL denote the downlink and uplink, respectively.

\section{Conclusion}

This paper addresses techniques to emulate bidirectional channel models in MPAC setups. A general framework for emulating bidirectional spatial channels for both TDD and FDD communication systems is proposed for arbitrary uplink and downlink probe configurations. Large scale and small scale parameters are modeled as identical in uplink and downlink channels, based on an extensive literature review. Fading coefficients are modeled as identical as well for TDD systems to achieve channel reciprocity. For FDD systems, there might be cases, where frequency separation is small and/or the FCF decreases very slowly, resulting in correlated uplink and downlink channels. The proposed algorithm, which basically attempts to generate uplink channels based on downlink channels in the MPAC, works well in both simulations and measurements. Results have demonstrated that, for a symmetric downlink and uplink probe configuration, channel reciprocity (i.e., fully correlated uplink and downlink channel) for TDD systems and desired arbitrary fading correlation between uplink and downlink channel can be achieved for FDD systems, whereas, for asymmetric probe configurations, the maximal achievable fading correlations between uplink and downlink channels, though generally high, are limited.

\section{Conflict of Interests}

The authors declare that there is no conflict of interests regarding the publication of this paper.

\section{Acknowledgments}

This work has been supported by the Danish National Advanced Technology Foundation via the Virtuoso project. The authors also appreciate the assistance from colleagues in 
Anite Telecoms Oy, Finland, especially Lic.Tech. Jukka-Pekka Nuutinen, now with Intel Mobile Communications.

\section{References}

[1] A. J. Paulraj, D. A. Gore, R. U. Nabar, and H. Bölcskei, "An overview of MIMO communications-a key to gigabit wireless," Proceedings of the IEEE, vol. 92, no. 2, pp. 198-217, 2004.

[2] M. A. Jensen and J. W. Wallace, "A review of antennas and propagation for MIMO wireless communications," IEEE Transactions on Antennas and Propagation, vol. 52, no. 11, pp. 28102824, 2004.

[3] 3GPP, "Verification of radiated multi-antenna reception performance of user equipment," 3GPP TR 37.977 V1.0.0, 2013.

[4] Y. Jing, X. Zhao, H. Kong, S. Duffy, and M. Rumney, "Twostage over-the-air (OTA) test method for LTE MIMO device performance evaluation," International Journal of Antennas and Propagation, vol. 2012, Article ID 572419, 6 pages, 2012.

[5] W. Yu, Y. Qi, K. Liu, Y. Xu, and J. Fan, "Radiated two-stage method for LTE MIMO user equipment performance evaluation," IEEE Transactions on Electromagnetic Compatibility, pp. $1-6,2014$.

[6] M. A. García-Fernández, J. F. Valenzuela-Valdés, and D. A. Sánchez-Hernández, "Latest advances in mode-stirred reverberation chambers for MIMO OTA evaluation of wireless communications devices," in Proceedings of the 5th European Conference on Antennas and Propagation (EUCAP'11), pp. 24272431, Rome, Italy, April 2011.

[7] P.-S. Kildal, C. Orlenius, and J. Carlsson, "OTA testing in multipath of antennas and wireless devices with MIMO and OFDM," Proceedings of the IEEE, vol. 100, no. 7, pp. 2145-2157, 2012.

[8] P. Kysti, T. Jämsä, and J.-P. Nuutinen, "Channel modelling for multiprobe over-the-air MIMO testing," International Journal of Antennas and Propagation, vol. 2012, Article ID 615954, 11 pages, 2012.

[9] W. Fan, F. Sun, P. Kyösti et al., "3D channel emulation in multiprobe setup," Electronics Letters, vol. 49, no. 9, pp. 623-625, 2013.

[10] R. K. Sharma, W. Kotterman, M. H. Landmann et al., "Over-theair testing of cognitive radio nodes in a virtual electromagnetic environment," International Journal of Antennas and Propagation, vol. 2013, Article ID 945283, 16 pages, 2013.

[11] A. Krah, M. Grimm, N. Murtaza et al., "Over-the-air test strategy and testbed for cognitive radio nodes," in Proceedings of the 30th URSI General Assembly and Scientific Symposium, pp. 1-4, IEEE, Istanbul, Turkey, August 2011.

[12] C. S. Park, Y.-P. E. Wang, G. Jöngren, and D. Hammarwall, "Evolution of uplink MIMO for LTE-advanced," IEEE Communications Magazine, vol. 49, no. 2, pp. 112-121, 2011.

[13] R. K. Sharma, C. Schneider, W. Kotterman et al., "Over-the-air testing of car-to-car and car-to-infrastructure communication in a virtual electromagnetic environment," in Proceedings of the 39th Annual Conference of the IEEE Industrial Electronics Society (IECON '13), pp. 6897-6902, IEEE, Vienna, Austria, November 2013.

[14] J.-P. Nuutinen, P. Kyösti, Y. Gao, and M. D. Foegelle, "On the MIMO OTA test system," in Proceedings of the 5th International ICST Conference on Communications and Networking in China (ChinaCom '10), pp. 1-5, IEEE, Beijing, China, August 2010.

[15] "Spatial channel model for Multiple Input Multiple Output (MIMO) simulations (Release 11)," 3GPP/3GPP2, TR 25.996 V11.0.0, 2012.
[16] P. Kyösti, J. Meinilä, L. Hentilä et al., "Winner II channel models," 2008.

[17] D. S. Baum, J. Salo, G. D. Galdo, M. Milojevic, P. Kyösti, and J. Hansen, "An interim channel model for beyond-3G systems: extending the 3GPP spatial channel model (SCM)," in Proceedings of the IEEE 61st Vehicular Technology Conference (VTC Spring '05), vol. 5, pp. 3132-3136, Stockholm, Sweden, May 2005.

[18] R. Zhang, Y. Zhang, Z. Zhong, and S. X. Lu, “The correlation properties of subchannel fading for non-continuous carrier aggregation based on indoor ultra-wideband measurement," in Proceedings of the IEEE Global Communications Conference (GLOBECOM '12), pp. 4071-4077, IEEE, December 2012.

[19] A. Kammoun, H. Khanfir, Z. Altman, M. Debbah, and M. Kamoun, "Preliminary results on 3D channel modeling: from theory to standardization," IEEE Journal on Selected Areas in Communications, vol. 32, no. 6, pp. 1219-1229, 2014.

[20] H. Omote, Y. Sugita, Y. Ohta, and T. Fujii, "Carrier frequency characteristic of Time-Spatial profile in outdoor LOS environments," in Proceedings of the 6th European Conference on Antennas and Propagation (EuCAP '12), pp. 423-427, IEEE, Prague, Czech Republic, March 2012.

[21] T. Fujii, Y. Ohta, and H. Omote, "Empirical time-spatial propagation model in outdoor NLOS environments for wideband mobile communication systems," in Proceedings of the IEEE 69th Vehicular Technology Conference (VTC Spring '09), pp. 1-5, IEEE, Barcelona, Spain, April 2009.

[22] J. Israel, G. Carrie, and M. A. Ighil, "A wideband and multifrequency propagation channel simulation," in Proceedings of the 7 th European Conference on Antennas and Propagation (EuCAP '13), pp. 677-680, Gothenburg, Sweden, April 2013.

[23] L. Bigler, H. P. Lin, S. S. Jeng, and G. Xu, "Experimental direction of arrival and spatial signature measurements at $900 \mathrm{MHz}$ for smart antenna systems," in Proceedings of the IEEE 45th Vehicular Technology Conference, vol. 1, pp. 55-58, IEEE, July 1995.

[24] H. Omote and T. Fujii, "Empirical arrival angular profile prediction formula for mobile communication systems," in Proceedings of the IEEE 65th Vehicular Technology Conference (VTC '07Spring), pp. 599-603, April 2007.

[25] K. Hugl, K. Kalliola, and J. Laurila, "Spatial reciprocity of uplink and downlink radio channels in FDD systems," COST273 $\mathrm{TD}(02) 066,2002$.

[26] S. Sesia, I. Toufik, and M. Baker, LTE: The UMTS Long Term Evolution, Wiley Online Library, 2009.

[27] Z. Shen, A. Papasakellariou, J. Montojo, D. Gerstenberger, and F. Xu, "Overview of 3GPP LTE-advanced carrier aggregation for $4 \mathrm{G}$ wireless communications," IEEE Communications Magazine, vol. 50, no. 2, pp. 122-130, 2012.

[28] C.-X. Wang, M. Pätzold, and Q. Yao, "Stochastic modeling and simulation of frequency-correlated wideband fading channels," IEEE Transactions on Vehicular Technology, vol. 56, no. 3, pp. 1050-1063, 2007.

[29] R. J. C. Bultitude, "Estimating frequency correlation functions from propagation measurements on fading radio channels: a critical review," IEEE Journal on Selected Areas in Communications, vol. 20, no. 6, pp. 1133-1143, 2002.

[30] T. Klingenbrunn and P. Mogensen, "Modelling frequency correlation of fast fading in frequency hopping GSM link simulations," in Proceedings of the 50th Fall IEEE VTS Vehicular Technology Conference (VTC '99), vol. 4, pp. 2398-2402, Amsterdam, The Netherlands, September 1999. 
[31] J. Nielsen, B. Yanakiev, S. Del Barrio, and G. Pedersen, "On antenna design objectives and the channel capacity of MIMO handsets," IEEE Transactions on Antennas and Propagation, vol. 62, no. 6, pp. 3232-3241, 2014.

[32] J. O. Nielsen, J. B. Andersen, P. C. F. Eggers, G. F. Pedersen, K. Olesen, and H. Suda, "Measurements of indoor $16 \times 32$ wideband MIMO channels at $5.8 \mathrm{GHz}$," in Proceedings of the 8th IEEE International Symposium on Spread Spectrum Techniques and Applications, pp. 864-868, IEEE, Sydney, Australia, AugustSeptember 2004.

[33] W. Fan, X. de Lisbona, F. Sun, J. Nielsen, M. Knudsen, and G. Pedersen, "Emulating spatial characteristics of MIMO channels for OTA testing," IEEE Transactions on Antennas and Propagation, vol. 61, no. 8, pp. 4306-4314, 2013.

[34] B. O. Hogstad, C. A. Gutiérrez, M. Pätzold, and P. M. Crespo, "Classes of sum-of-cisoids processes and their statistics for the modeling and simulation of mobile fading channels," EURASIP Journal on Wireless Communications and Networking, vol. 2013, no. 1, pp. 1-15, 2013.

[35] G. H. Golub and C. F. Van Loan, Matrix Computations, vol. 3, JHU Press, 2012.

[36] A. Khatun, T. Laitinen, V.-M. Kolmonen, and P. Vainikainen, "Dependence of error level on the number of probes in overthe-air multiprobe test systems," International Journal of Antennas and Propagation, vol. 2012, Article ID 624174, 6 pages, 2012. 

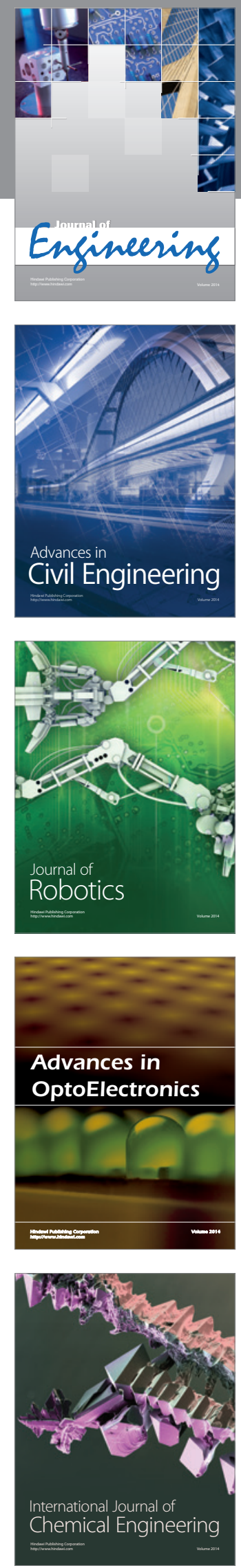

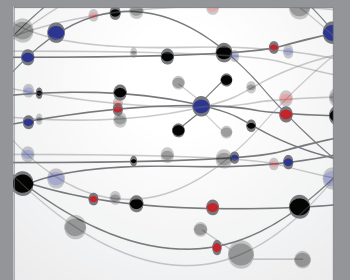

The Scientific World Journal
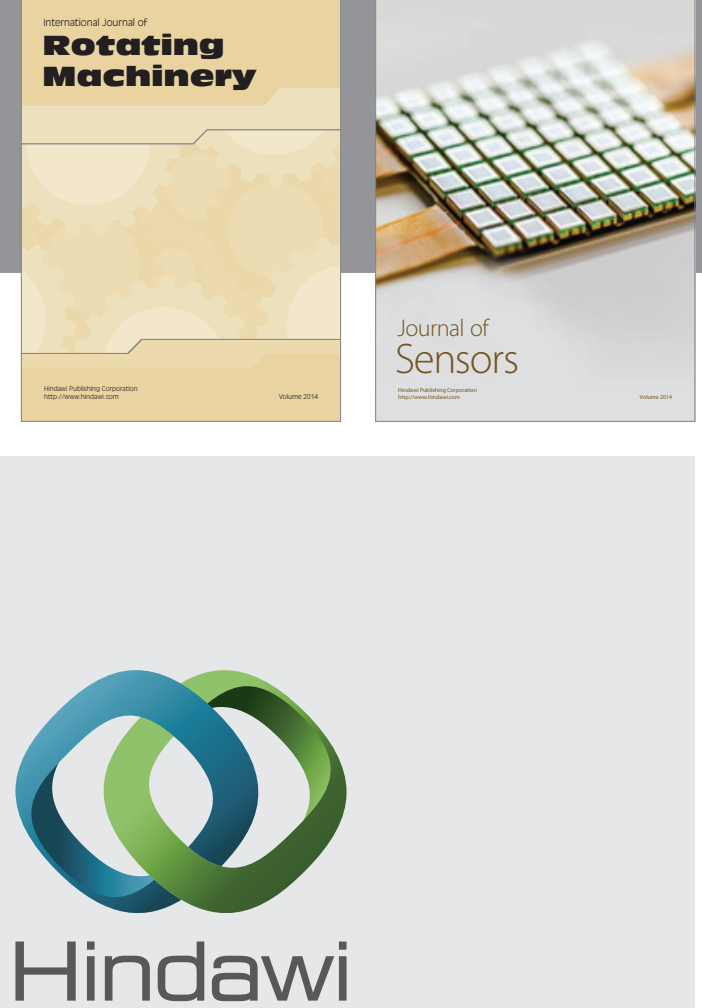

Submit your manuscripts at http://www.hindawi.com
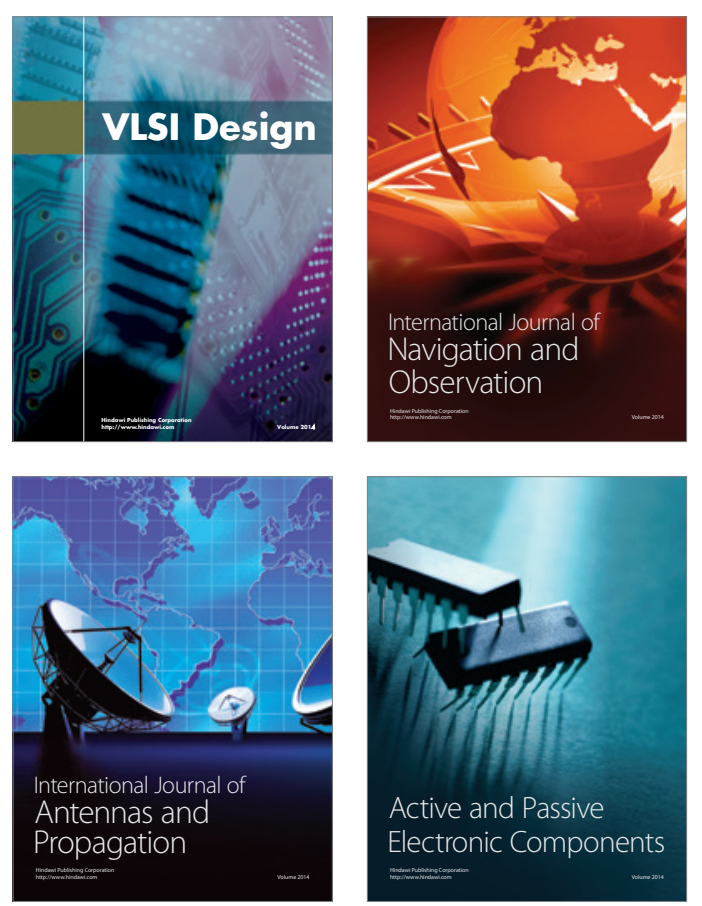
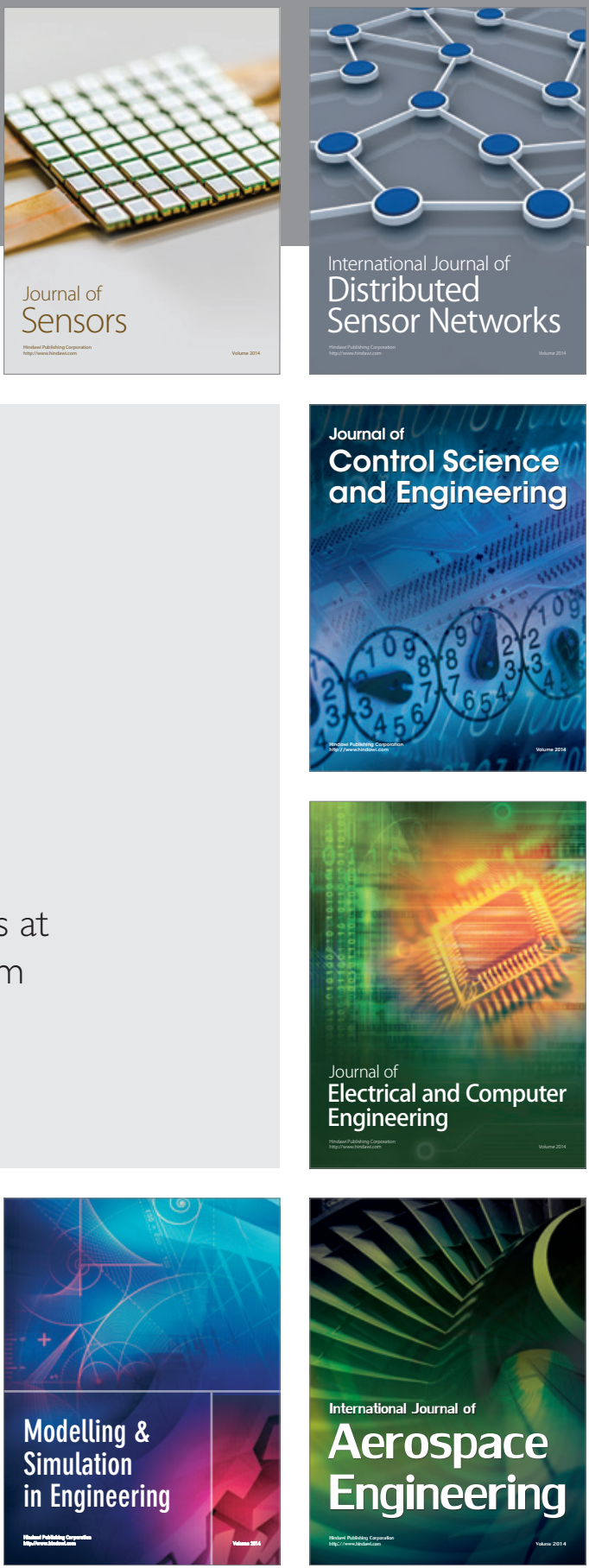

Journal of

Control Science

and Engineering
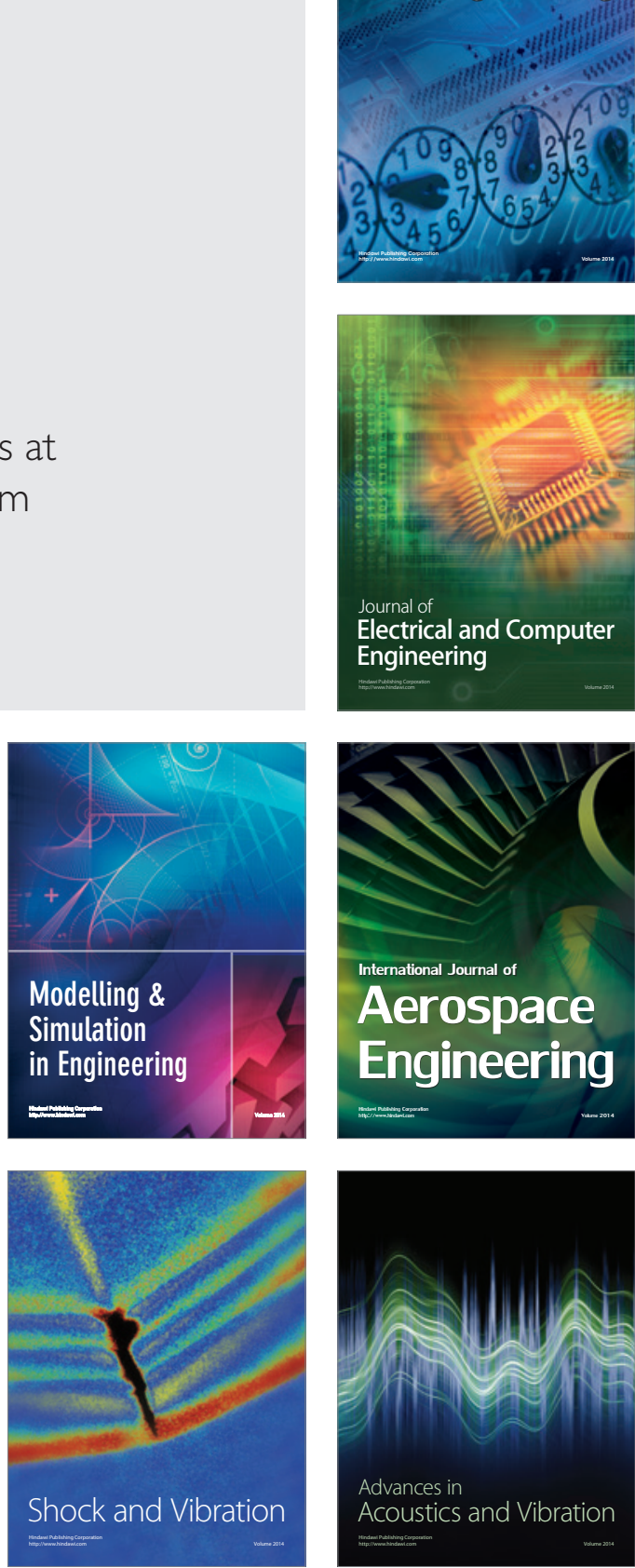